\title{
A high-resolution oxygen A-band spectrometer (HABS) and its radiation closure
}

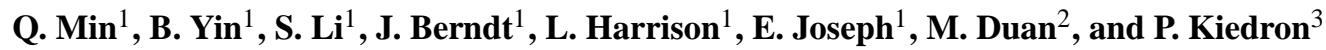 \\ ${ }^{1}$ Atmospheric Science Research Center, State University of New York, Albany NY 12203, USA \\ ${ }^{2}$ LAGEO, Institute of Atmospheric Physics, Chinese Academy of Sciences, Beijing, China \\ ${ }^{3}$ Cooperative Institute for Research in Environmental Sciences, University of Colorado, Boulder, Colorado, USA
}

Correspondence to: Q. Min (qmin@albany.edu)

Received: 17 January 2014 - Published in Atmos. Meas. Tech. Discuss.: 5 February 2014

Revised: 23 April 2014 - Accepted: 25 April 2014 - Published: 17 June 2014

\begin{abstract}
Various studies indicate that high-resolution oxygen A-band spectrum has the capability to retrieve the vertical profiles of aerosol and cloud properties. To improve the understanding of oxygen A-band inversions and utility, we developed a high-resolution oxygen A-band spectrometer (HABS), and deployed it at Howard University Beltsville site during the NASA Discover Air-Quality Field Campaign in July, 2011. By using a single telescope, the HABS instrument measures the direct solar and the zenith diffuse radiation subsequently. HABS exhibits excellent performance: stable spectral response ratio, high signal-to-noise ratio (SNR), high-spectrum resolution $(0.016 \mathrm{~nm})$, and high out-of-band rejection $\left(10^{-5}\right)$. For the spectral retrievals of HABS measurements, a simulator is developed by combining a discrete ordinates radiative transfer code (DISORT) with the High Resolution Transmission (HITRAN) database HITRAN2008. The simulator uses a double- $k$ approach to reduce the computational cost. The HABS-measured spectra are consistent with the related simulated spectra. For directbeam spectra, the discrepancies between measurements and simulations, indicated by confidence intervals $(95 \%)$ of relative difference, are $(-0.06,0.05)$ and $(-0.08,0.09)$ for solar zenith angles of 27 and $72^{\circ}$, respectively. For zenith diffuse spectra, the related discrepancies between measurements and simulations are $(-0.06,0.05)$ and $(-0.08,0.07)$ for solar zenith angles of 27 and $72^{\circ}$, respectively. The main discrepancies between measurements and simulations occur at or near the strong oxygen absorption line centers. They are mainly due to two kinds of causes: (1) measurement errors associated with the noise/spikes of HABS-measured spectra, as a result of combined effects of weak signal, low SNR,
\end{abstract}

and errors in wavelength registration; (2) modeling errors in the simulation, including the error of model parameters setting (e.g., oxygen absorption line parameters, vertical profiles of temperature and pressure) and the lack of treatment of the rotational Raman scattering. The high-resolution oxygen A-band measurements from HABS can constrain the active radar retrievals for more accurate cloud optical properties (e.g., cloud optical depth, effective radius), particularly for multi-layer clouds and for mixed-phase clouds.

\section{Introduction}

Oxygen A-band $(759-770 \mathrm{~nm})$ is one of the most prominent near-infrared features in the atmospheric spectrum. It has been studied extensively for remote sensing from satellite measurements to ground-based measurements (Grechko et al., 1973; Mitchell and O'Brien, 1987; Fischer and Grassl, 1991; Fischer et al., 1991; O’Brien and Mitchell, 1992; Harrison and Min, 1997; Pfeilsticker et al., 1998; Veitel et al., 1998; Stam et al., 1999; Min and Harrison, 1999, 2004; Portmann et al., 2001; Koelemeijer et al., 2001; Min et al., 2001, 2004a; Min and Clothiaux, 2003; Kokhanovsky et al., 2006a, b; Boesche et al., 2006, 2009; Li and Min, 2010, 2013). Oxygen is a well-mixed gas in the atmosphere; the pressure dependence of oxygen A-band absorption line parameters provides a practical way for retrieving photon path length distributions and vertical information of aerosols and clouds (Harrison and Min, 1997; Pfeisticker et al., 1998; Veitel et al., 1998; Min and Harrison, 1999, 2004; Portmann et al., 2001; Min et al., 2004a; etc.). However, except for a 
few sensors, such as Fourier transform spectrometer (FTS) in the Greenhouse gases Observing SATellite (GOSAT, Kuze et al., 2009), most of routine measurements of oxygen Aband spectra are from low- or moderate-resolution spectrometers. As discussed in Min and Clothiaux (2003), the low- or moderate-resolution oxygen A-band spectra only can provide two independent retrieved parameters of photon path length distribution.

In principle, high-resolution oxygen A-band spectra can discriminate atmospheric scattering from surface scattering, and thus provide a better remote-sensing technique to retrieve cloud and aerosol macrophysical/microphysical properties (Stephens and Heidinger, 2000; Heidinger and Stephens, 2000, 2002; Min and Harrison, 2004; Stephens et al., 2005). As stated in Min and Harrison (2004), with highresolution oxygen A-band measurements, four or five independent pieces of information can be obtained, depending on the instrument resolution, the out-of-band (OOB) rejection, and the Signal-to-Noise Ratio (SNR).

To understand oxygen A-band inversions and utility, we developed a high-resolution oxygen A-band spectrometer (HABS) with polarization capability. It was deployed at Howard University Beltsville Campus (HUBC) in Maryland during the NASA Discover Air-Quality Field Campaign in July, 2011. This paper specifically reports on the development of the HABS and its spectral performance. We evaluate the spectral performance of HABS through radiation closure by comparing HABS-measured spectra with simulated spectra. The simulation of high-resolution oxygen A-band spectrum is based on our HABS simulator that combines the discrete ordinates radiative transfer (DISORT) model (Stamnes et al., 1988) with the High Resolution Transmission (HITRAN) database HITRAN2008 (Rothman et al., 2009). More importantly, all key model inputs are based on the collocated measurements during the field campaign. In particular, aerosol optical properties are derived from a Multi-Filter Rotating Shadowband Radiometer (MFRSR) and a Raman lidar. MFRSR provides measurements of spectral solar radiation and retrieved aerosol and cloud optical properties (Min and Harrison, 1996; Min et al., 2003, 2004b, c, 2008). Raman lidar has the ability to measure the vertical profiles of aerosol scattering ratio at high time resolution and height resolution (Connell et al., 2009).

This paper is organized as follows. Section 2 provides the instrument design and its characteristics and performance; Sect. 3 describes a fast and accurate HABS simulator and HABS measurement simulations; Sect. 4 discusses the comparisons of HABS measurements with model simulations for radiation closure; Sect. 5 elaborates the potential applications of HABS and our research plan about it in the near future; Sect. 6 provides a brief summary and some concluding remarks.

\section{Instrument design and its performance}

\subsection{Instrument design}

The HABS, shown in Fig. 1, was developed by the Atmospheric Science Research Center (ASRC) of State University of New York at Albany. It consists of an altazimuth tracker fore-optics, a high-resolution grating monochromator, a high-performance charge-coupled device (CCD) assembly, and a temperature controlling system.

A sketch of the HABS optics is shown in Fig. 2. Through a telescope with a field of view (FOV) of $2.71^{\circ}$ and a pair of total reflection prisms, the elevation-azimuth sun tracker can track the sun or point to the zenithal direction automatically. This fore-optic enables to measure both direct beam and diffuse radiances using the same system. Since the photon path length distribution is known for direct beam radiance (e.g., a $\delta$ function), the direct beam measurements can be used to assess instrument features and absorption line parameters with simple Beer's law. The direct beam measurements can also be used to directly construct the retrieval kernels (Rodgers, 2000; Min and Clothiaux, 2003). The well-tested instrument functions and line parameters or the constructed retrieval kernels from direct beam measurements are readily used for the retrievals from diffuse radiance measurements.

The grating monochromator consists of a high line-density grating (1800 grooves per $\mathrm{mm})$ and two long focal-length mirrors with focal lengths of 32 inches and 38 inches. It provides high-resolution spectrally resolved direct-beam and zenith diffuse radiance on a CCD array $(1024 \times 256)$, covering the entire oxygen A-band from 759 to $769 \mathrm{~nm}$. The spectrometer is equipped with a high performance CCD camera (Horiba Scientific Synapse $1024 \times 256$ CCD detector). The CCD area of the system is thermoelectrically cooled to $-70{ }^{\circ} \mathrm{C}$ to reduce the readout noise. The $\mathrm{CCD}$ array works at two modes: (1) image mode for grating spectrometer assembling; and (2) spectrum mode for the instrument performance testing (Sect. 2.2) and solar radiation measurements (Sect. 2.3), in which every column of pixels are combined to one pixel and the CCD array works as a linear CCD $(1024 \times 1)$. The whole optical system is housed in an enclosure to maintain temperature stability and to protect it from the weather. As a high-resolution spectrometer, the instrument is sensitive to the environment temperature, which can result in the spectrum wavelength shifting (Platt and Stutz, 2008; Li and Min, 2012). Because each pixel measures different portions of the absorption spectrum, a shifting wavelength mapping of the spectrum will bring in errors to the retrieval processes. In order to alleviate these errors, a temperature controlling system is implemented into the instrument, which consists of a temperature controller, temperature sensors, fans, heaters, and a water cooling subsystem (not shown here). This temperature controlling system can keep the HABS inner temperature stable within $0.1^{\circ} \mathrm{C}$. It helps 


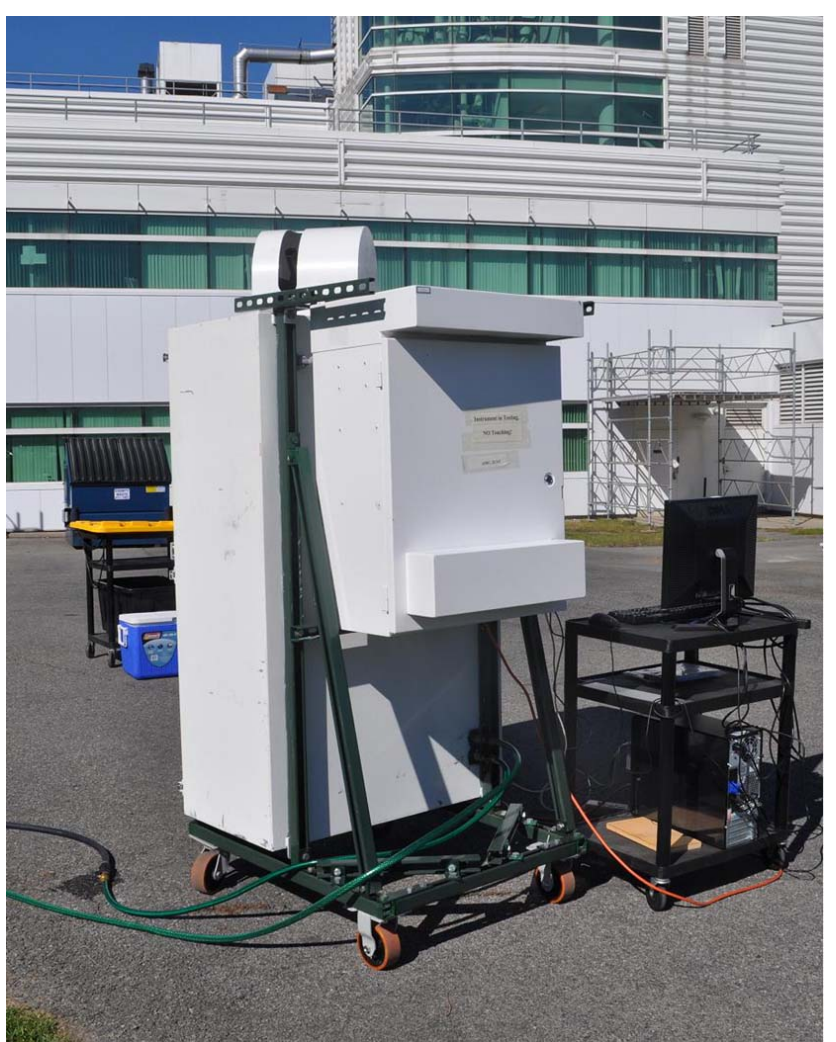

Figure 1. The photo of the HABS taken at the Atmospheric Science and Research Center, State University of New York at Albany during the field observation.

to restrain the spectrum shifting and protect the instrument components from outside temperature variations.

In the fore-optic module of HABS, two filters are used to suppress radiation outside the band, and an electronic shutter is used to control the time of exposure. A filter wheel in the fore-optic enables six modes: open mode, diffuser mode, and four polarizer modes with different orientations (i.e., 0 , 45, 90, and $135^{\circ}$ ), as shown in Fig. 2. The diffuser mode is used for direct-beam measurements, to reduce the signal and fill up the slit of the spectrograph. Due to the small FOV $\left(2.71^{\circ}\right)$ of the instrument, if no cloud (or only very thin clouds) exists in the sun-sensor direction, the diffuse photon is ignorable compared to the sun direct beam. The open mode and four polarizer modes are used for zenith diffuse measurements. In the open mode, the incident light can pass through the filter wheel directly without any optical components. The polarizer modes enable to measure the degree of polarization in the atmosphere, which enhances retrieval capability of oxygen A-band spectroscopy. As the grating and mirrors have polarization-dependent reflectivity properties, neglect of such an instrument's polarization sensitivity can lead to errors of several tens of percent in the values of radiance measured at wavelengths where the instrument's polarization sensitivity is highest (Natraj et al., 2007; Stam and

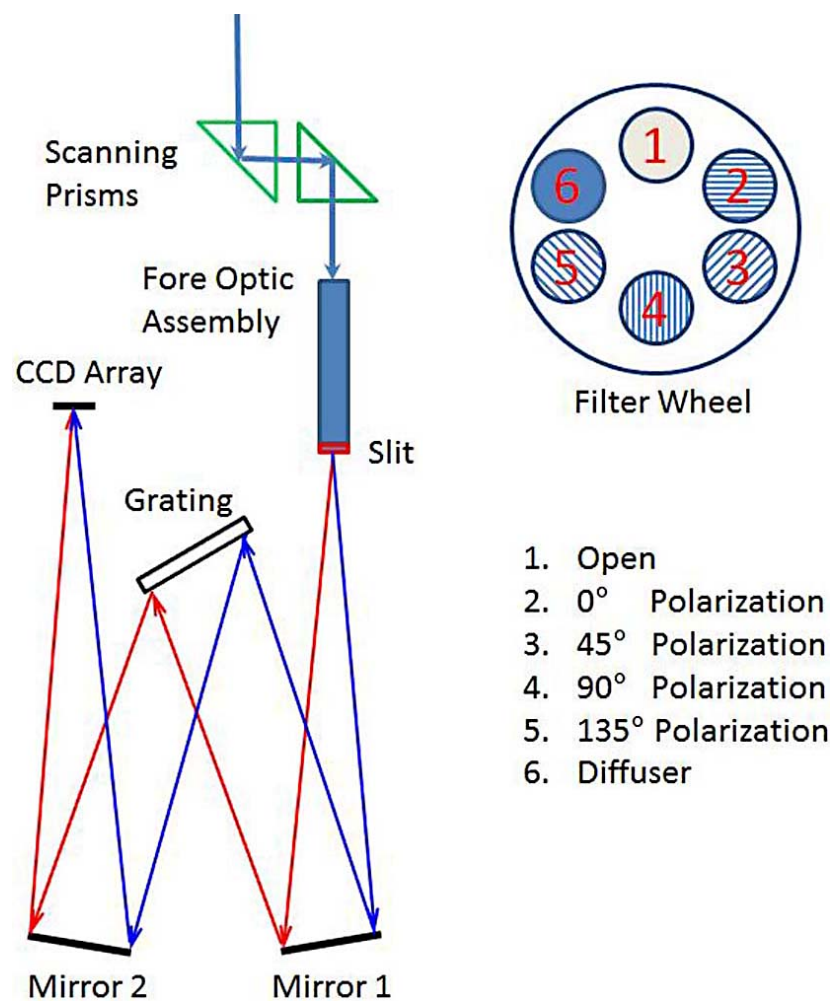

Figure 2. Optical setup of the high-resolution oxygen A-band spectrometer (HABS).

Hovenier, 2005; Levy et al., 2004; Schutgens and Stammes, 2003; Oikarinen, 2001; Lacis, 1998; Mishchenko et al., 1994; Charles and Kattawar, 1994).

The four polarizer modes can be used to derive the degree of polarization (DOP) for the oxygen A-band spectra, through Eqs. (1)-(5) (Berry et al., 1977; McMaster, 1954, 1961):

$$
\begin{aligned}
& I=I\left(0^{\circ}\right)+I\left(90^{\circ}\right)=<\left|E_{x}\right|^{2}+\left|E_{y}\right|^{2}> \\
& Q=I\left(0^{\circ}\right)-I\left(90^{\circ}\right)=<\left|E_{x}\right|^{2}-\left|E_{y}\right|^{2}> \\
& U=I\left(45^{\circ}\right)-I\left(135^{\circ}\right)=R e<E_{x} E_{y}> \\
& V=0=I m<E_{x} E_{y}> \\
& \text { DOP }=\left(Q^{2}+U^{2}+V^{2}\right)^{1 / 2} / I,
\end{aligned}
$$

where we choose a Cartesian coordinate system $(x, y, z)$ to indicate the light propagation. $E_{x}$ and $E_{y}$ are the electric field components in the $x$ and $y$ directions, perpendicular to the direction of light propagation $z . I\left(0^{\circ}\right), I\left(45^{\circ}\right), I\left(90^{\circ}\right)$, and $I\left(135^{\circ}\right)$ are HABS-measured polarized radiances under different polarization modes. $I, Q, U$ and $V$ are the four 


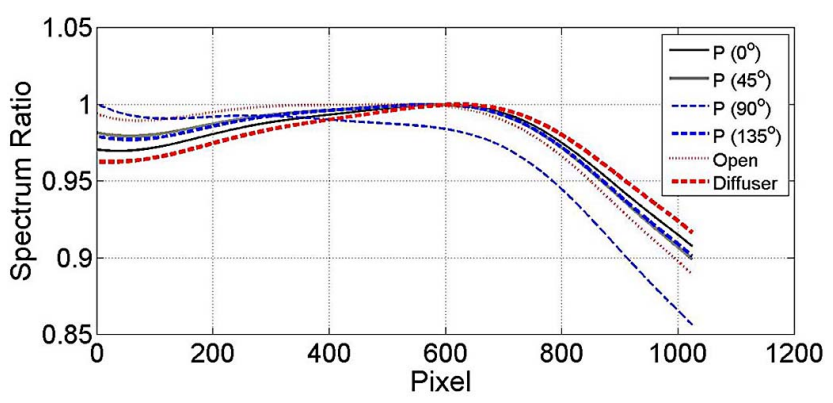

Figure 3. Spectral response for different optical channels (i.e., open, diffuser, and 4 polarizers with different orientations) measured with the Gamma Scientific's Model 5000 FEL 1000-Watt lamp source.

Stokes parameters (Van de Hulst, 1957), in which V component is 0 based on current instrument measurements (Eq. 4); and DOP is the derived degree of polarization. The measurement of polarization of oxygen A-band spectra improves the retrieval ability for aerosols and ice clouds (Hansen and Travis, 1974; Bréon and Colzy, 2000; Deuzé, et al., 2000, 2001; Chowdhary et al., 2001, 2005; etc.).

\subsection{Instrument performance}

As stated previously, before the incident light arrives at the entrance slit of grating spectrometer, it passes through a pair of prisms, two bandpass filters, and one of the filter wheel channels. This modulates the spectral shape of the incident light, which can be presented by a "filter function". To obtain the response of the overall filter function, we use the Gamma Scientific's Model 5000 FEL 1000-Watt lamp source to provide the reference spectrum. The spectral response ratios of HABS-measured spectrum and the reference spectrum at different channels of filter wheel are shown in Fig. 3. In this study, these response functions are measured many times during long-term in-lab experiments and field observations, and they changed very little, suggesting very stable performance of the HABS. The spectral response ratios are crucial for the recovery of modulated measured spectral shape, which impacts the accuracy of the spectral intensity and normalization directly.

As discussed in Min and Harrison (2004), the slit function is crucial for evaluation of spectrometer performances. The slit function of the HABS was once obtained using an Ar lamp $(763.511 \mathrm{~nm})$ and used for wavelength registration study (Li and Min, 2012). However, some contamination of other weak emission lines of the Ar lamp may contribute to the stray light of the slit function. In this study, we used a laser diode DFB-763 $(763.0 \mathrm{~nm})$ to measure the slit function. As shown in Fig. 4, the full width at half maximum (FWHM) is about 1.55 pixels or $0.016 \mathrm{~nm}$ (about $0.26 \mathrm{~cm}^{-1}$ at oxygen A-band), which is narrower than the Ar lamp measured one (i.e., 1.85 pixels or $0.019 \mathrm{~nm}$ or $0.30 \mathrm{~cm}^{-1}$ ). The OOB rejection (at about $5 \mathrm{~nm}$ distance from the slit function maximum)

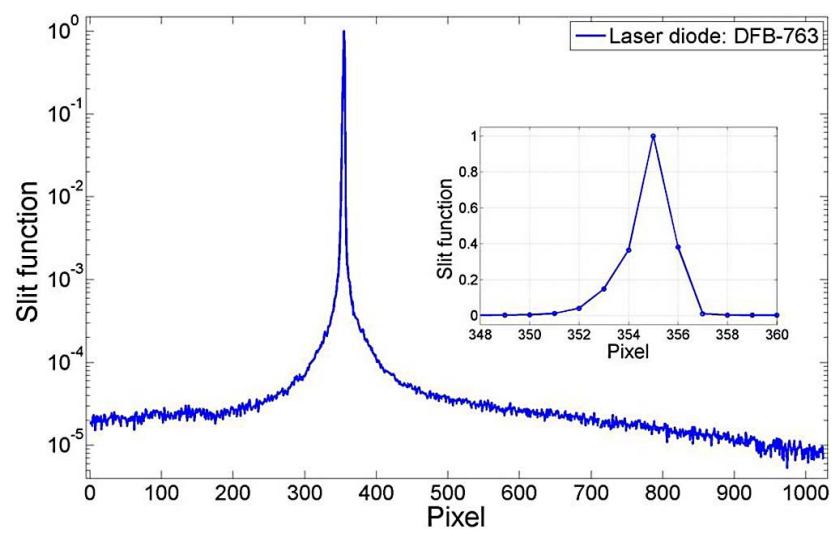

Figure 4. Slit function of high-resolution oxygen A-band spectrometer (HABS): the insert indicate a FWHM resolution of 1.55 detector pixels.

is about $10^{-5}$, similar to the Ar lamp measurement. Similar to the spectral response ratios, the slit function changed very little during the long-term experiments. The slit function is vital important for the simulation of instrument measurements (see Sect. 3).

The SNR is determined by both read-out and photon electron shot noise, the latter being Poisson distributed. When the signal is weak, such as the signal near a strong absorption line center, the read-out noise (and Poisson noise of dark signal) dominates. Due to the low temperature (about $-70^{\circ} \mathrm{C}$ ) of the CCD module, the standard deviation (SD) of readout noise for CCD pixels is less than 5 counts, and their mean value is about 3 counts. Figure 5 shows one case of the SNR that measured at solar zenith angle (SZA) of $31^{\circ}$. It is estimated based on 24 consecutive measurements, in which each exposure is $0.25 \mathrm{~s}$. This method assumes the reference source (solar direct beam) is constant during this short period $(<10 \mathrm{~s})$. By comparing the mean measurement value and the standard deviation of measurement noise, the SNR can be obtained. The minimum SNR is 78 and 111 in R (left) and P (right) branches (Spiering et al., 2010), respectively. The signal strength depends on solar zenith angle and sky conditions, so does the SNR. To obtain the best SNR, the exposure time is automatically adjusted based on the strength of incident solar radiation. It is implemented by taking two measurements consequently: (1) the first measurement, which only takes very short exposure time to estimate the strength of incident solar radiation; (2) the second measurement, measured with the optimal exposure time estimated from the amplitude of the first measurement. Furthermore, to remove the detector offset caused by dark current, every time before the solar radiation measurement, we will measure the dark current firstly. Subtracting the dark current from the original measured solar spectrum, we obtain the exact signal of measured solar spectrum. 


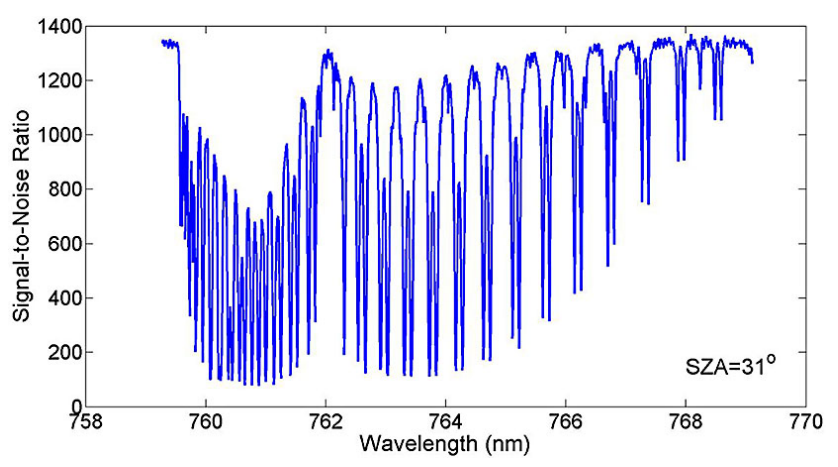

Figure 5. Estimated signal-to-noise ratio (SNR) for a direct spectrum taken at Howard University Beltsville site at 19:13 UTC on 14 June 2011.

In this spectrometer the FWHM of the slit function is $0.016 \mathrm{~nm}$, thus measurements of sharply structured absorption features are very sensitive to small spectral shifts. For HABS, the impact of wavelength shift has been extensively discussed by Li and Min (2012). Even if a very small wavelength shift (e.g., $0.001 \mathrm{~nm}$ ) occurs, the distribution of oxygen absorption optical depth contributing to the absorption line center will change significantly. To reduce the error induced by the wavelength shift, Li and Min (2012) developed a self-calibrating wavelength registration method. This method can accurately register each measured spectrum individually and fast, with a wavelength registration error that is within $0.0001 \mathrm{~nm}$. In this study, we used this method to make wavelength registration.

\subsection{HABS measurements}

We deployed the HABS at HUBC during the NASA Discover Air-Quality Field Campaign in July, 2011. During this field campaign, many instruments (e.g., MFRSR, Raman lidar, sonde balloon, etc.) worked together to observe the air-quality, aerosol and cloud properties. Figure 6a shows HABS-measured oxygen A-band direct beam spectra and the related zenith diffuse spectra (at open mode) under clearday situations. Figure $6 \mathrm{~b}$ shows two HABS-measured oxygen A-band zenith diffuse spectra (at open mode) for thick clouds and cirrus clouds, respectively. It is clear that the primary absorption lines in $\mathrm{P}$ branch are resolved. It is worthy to mention that in Fig. 6 and other figures in the following sections, the spectra are represented as normalized radiance, which can better indicate the absorption characteristics in $\mathrm{O}_{2}$ A-band. The normalized radiance is calculated by dividing the original measured/simulated radiance with the spectrum baseline. The latter is derived by the measured/simulated radiance at the shoulders of the $\mathrm{O}_{2} \mathrm{~A}$-band (without oxygen absorption), for example, at about 759.4 and $768.8 \mathrm{~nm}$, which have the best SNR and are insensitive to spectrum shifting. They are only impacted by the column integrated
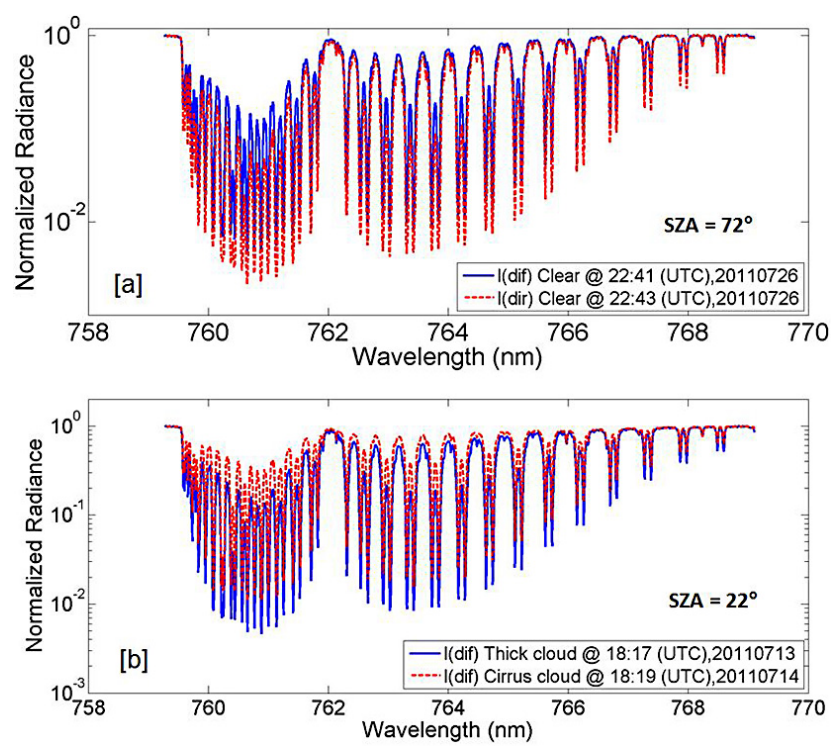

Figure 6. HABS-measured oxygen A-band spectra for the direct solar beam at $\mathrm{SZA}=72^{\circ}$ (a) and diffuse zenith spectra (b) under clear skies for $\mathrm{SZA}=22^{\circ}$ for different cloud optical depths.

atmospheric optical depth, and less sensitive to the vertical distribution of aerosol and cloud optical properties.

Figure 7 shows one case of the measured high-resolution oxygen A-band spectra of zenith diffuse radiation for four polarizations. It was measured at 13:08 UTC on 26 July, 2011. In this case, no cloud exists; SZA is $56^{\circ}$, and the MFRSR-derived AOD (at $760 \mathrm{~nm}$ ) is about 0.04 (Fig. 8b). Obviously, the spectra measured under polarization modes are smaller than under the open mode and vary significantly with polarizer orientations. The derived DOP spectrum, shown in Fig. 7b, exhibits polarization characteristics associated with oxygen absorption lines. As shown in Eqs. (1)-(5), DOP is derived through subtractions among four spectra. The residuals are extremely sensitive to the signal-to-noise ratio (SNR) of spectrometer. Weak signals and low SNRs at the strong absorption line centers could result in some noise in the DOP spectrum. Additionally, slight shifts of wavelength associated with different spectra could also result in spikes in the DOP spectrum. Again, those spikes are at or near the strong absorption line centers, as a result of the combined effect of weak signals, low SNRs, and slight wavelength shifts. The HABS polarization measurements will be evaluated, analyzed, and discussed in detail in a forthcoming paper.

\subsection{Simulation of high-resolution oxygen A-band spectra}

Analyzing the high-resolution spectra measurements and applying them to the retrievals of cloud and aerosol properties require forward radiative transfer calculations to simulate the observed spectra. In the model simulation, the 

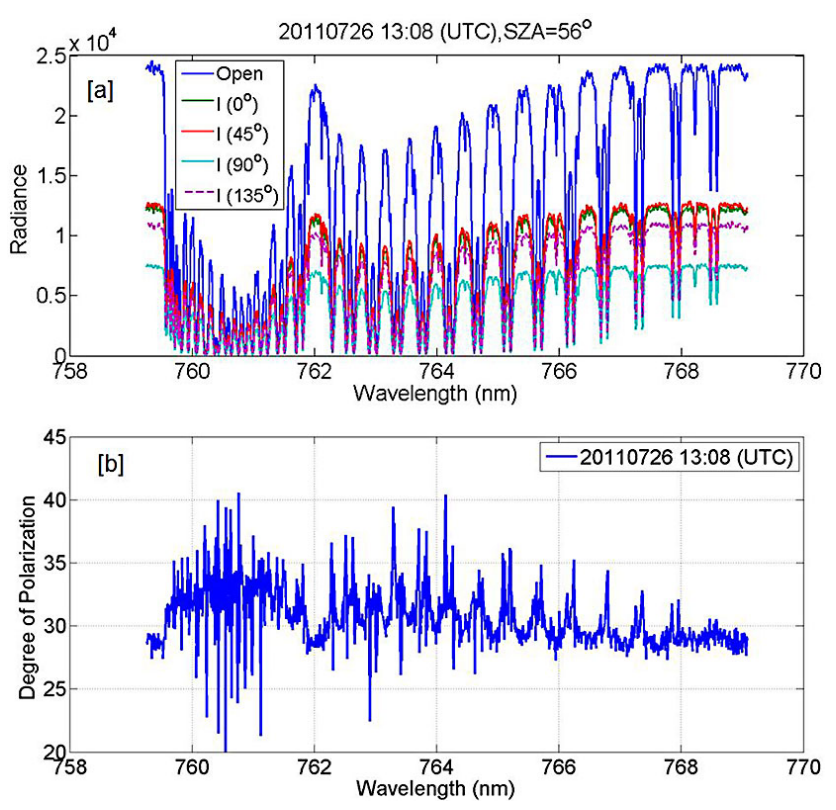

Figure 7. HABS-measured oxygen A-band spectra for the zenith diffuse radiation (a) and the related derived degree of polarization (DOP) spectrum (b) at 13:08 UTC on 26 July 2011. The radiance is indicated by the analog to digital converter (ADC) counts.

background atmospheric information (e.g., temperature, air pressure, aerosol, etc.) are from observation, and the highresolution solar reference spectrum at the top of the atmosphere (TOA) is provide by Chance and Kurucz (2010), which can be downloaded from the website of HarvardSmithsonian Center for Astrophysics (http://kurucz.harvard. edu/sun.html). For the measured spectra, each pixel contains spectral response of multiple absorption lines, convolving with the slit function. Therefore, to simulate HABSmeasured spectrum, we need to make radiative transfer calculations in a line-by-line domain. To complete these calculations, vertical profiles of oxygen absorption optical depth are required also in a line-by-line domain. In this study, the oxygen absorption profiles are originally calculated by the line-by-line radiative transfer model (LBLRTM 12.1) (Clough et al., 2005) based on the HITRAN database HITRAN2008. However, calculating oxygen absorption optical depth profiles with LBLRTM is very time-consuming (more than $5 \mathrm{~h}$ for the entire oxygen A-band by a fast PC). Because the atmospheric structure is always changing with time, frequent recalculation of the oxygen absorption optical depth profiles is required. To reduce the computational cost, it is necessary to develop a fast method to recalculate the oxygen absorption optical depth profiles accurately.

Based on the given background atmospheric information, the solar reference spectrum at TOA, and the recalculated oxygen absorption optical depth profiles, we can make radiative transfer calculations in a line-by-line domain. However, running a radiative transfer model in a line-by-line domain is also very time-consuming. To reduce the computational cost for operational retrievals, Duan et al. (2005) developed a fast radiative transfer model by using a double- $k$ distribution approach. Based on this fast radiative transfer model, we developed a HABS simulator to simulate the HABS measurement.

\subsection{Calculation of oxygen absorption optical depth profiles}

As stated previously, the oxygen absorption coefficients are determined by the atmosphere pressure and temperature. Chou and Kouvaris (1986) proposed that the absorption coefficients $(k)$ at wavenumber $(v)$ can be defined as a function of air pressure ( $p)$ and temperature (T) as shown in Eq. (6).

$$
\begin{aligned}
\ln (k(v, p, T)) & =a_{0}(v, p)+a_{1}(v, p) \times\left(T-T_{\text {mean }}\right) \\
& +a_{2}(v, p) \times\left(T-T_{\text {mean }}\right)^{2},
\end{aligned}
$$

where $a_{0}, a_{1}, a_{2}$ and $T_{\text {mean }}$ are previously calculated parameters by fitting three pairs of given $(p, T)$. In a similar way, the oxygen absorption optical depth $(\tau)$ can be also defined as a function of $p$ and $T$ for any atmospheric layer as shown in Eq. (7).

$$
\begin{aligned}
\ln (\tau(v, p, T)) & =a_{0}(v, p)+a_{1}(v, p) \times\left(T-T_{\text {mean }}\right) \\
& +a_{2}(v, p) \times\left(T-T_{\text {mean }}\right)^{2}
\end{aligned}
$$

In this study, all parameters of Eq. (7), that is, $a_{0}, a_{1}, a_{2}$ and $T_{\text {mean }}$, are derived by the results of three or more given atmospheric profiles. In the LBLRTM model, there are six typical geographic-seasonal model atmospheres: tropical model, mid-latitude summer model, mid-latitude winter model, subarctic summer model, subarctic winter model, and United States Standard (1976) model. Based on these six model atmospheres, six sets of oxygen absorption optical depth profiles are calculated in the line-by-line domain. Using three of them (e.g., tropical model, subarctic winter model, and United States Standard model), we derive the three parameters (i.e., $a_{0}, a_{1}, a_{2}$ ) in Eq. (7) for every wavenumber. These derived parameters are stored in a parameter database in the line-by-line domain. The oxygen absorption optical depth profiles for all the six model atmospheres have been recalculated through Eq. (7) with derived parameters and compared with the original LBLRTM calculations. The comparison indicates that the accuracy of the fast parameterization scheme is within $1 \%$ (not shown here) and the computing time is reduced to $0.2 \%$

\subsection{Double- $k$ distribution approach}

Based on the calculated oxygen absorption optical depth profiles and other observed or derived atmospheric properties (e.g., surface albedo, solar zenith angle, etc.), we use the DISORT model to calculate radiative transfer in the atmosphere. Although the LBLRTM model can be used to simulate the high-spectral-resolution radiance, it is very 

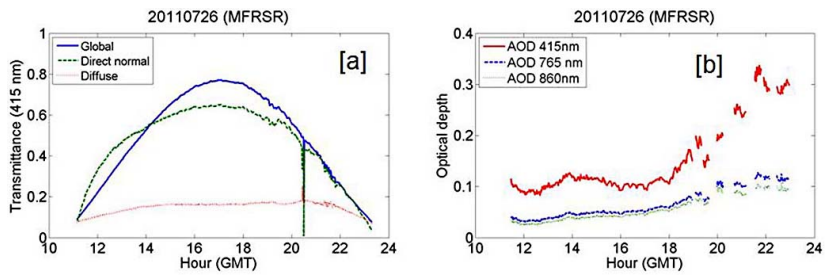

Figure 8. (a) Daily variation of direct normal, global horizontal and diffuse horizontal irradiances from MFRSR measurements on 26 July 2011 at Howard University Beltsville site. (b) Daily variation of derived aerosol optical depth at 415, 760 and $870 \mathrm{~nm}$ from MFRSR measurements.

time-consuming. A fast and accurate forward radiative transfer model is crucial to reduce the computational cost for operational retrievals. Min and Harrison (2004) outlined a $k$ distribution approach for simulating the oxygen A-band spectrum under clear-sky conditions. In their approach, the radiation from absorption and scattering processes of cloud and aerosol is split into the single- and multiple-scattering components: the first scattering component is computed accurately, and multiple scattering (second order and higher) radiance is calculated approximately. Duan et al. (2005) extended and modified this technique to all-sky conditions on the basis of the equivalent theorem with a double- $k$ distribution approach to account for the uncorrelated nature of overlapped absorption lines. In this double- $k$ approach, there are two integrated absorption optical depths: (1) the total absorption optical depth $(k)$, and (2) absorption optical depth from the top of the atmosphere to the scattering layer $\left(k_{0}\right)$. They are used together to account for the vertical distribution of gaseous absorption in multiple-scattering media. In this study, we use the DISORT code coupled with the double- $k$ distribution approach to simulate the oxygen A-band spectrum.

\subsection{HABS fitting model}

To simulate the HABS measurements, the model calculated high-resolution spectrum needs to be convolved with the instrument slit function. As stated previously, the measured HABS slit function shows high-performance spectral ability. However, its spectral resolution is much lower than the required spectral resolution of the forward model. To match the model calculated spectra, we interpolate the measured HABS slit function to the model resolution. At the slit function maximum, the choice of interpolating function has substantial impacts on the shape of the slit function ( $\mathrm{Li}$ and $\mathrm{Min}$, 2012). In this study, we used the same method as Li and Min (2012), which has been proven to have the ability to make an accurate fitting calculation: the shapes and wavelength positions of the absorption lines in simulated spectra and observed spectra are well consistent with each other.
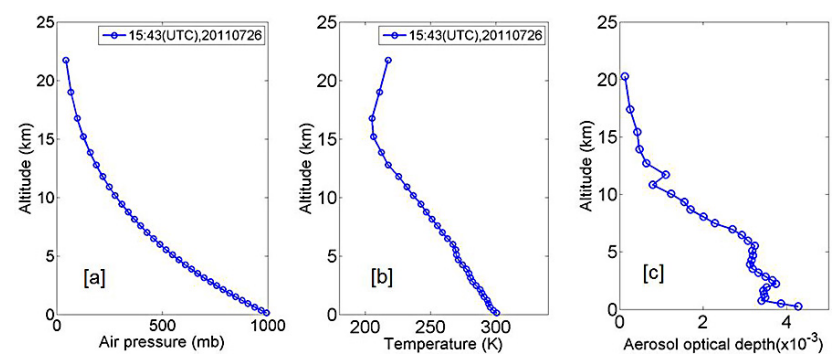

Figure 9. Profiles of (a) air pressure and (b) temperature from sonde balloon measurements at 15:43 UTC on 26 July 2011 at Howard University Beltsville site. (c) Aerosol optical depth profile estimated from Raman lidar-measured aerosol-scattering ratio profile normalized with MFRSR-derived AOD.

\section{Comparison of HABS measurements with model simulations}

As shown in Fig. 8a, MFRSR measurements indicate that 26 July 2011 was a clear day for radiation closure study of HABS. The vertical profiles of air pressure and temperature on that day were provided by the collocated sonde balloon measurements (shown in Fig. 9a and b). In the model simulation, to be consistent with the oxygen absorption optical depth profiles, the balloon sonde measured profiles are layered into 34 atmospheric layers, with an increment of air pressure of $30 \mathrm{mbar}$. The aerosol optical depth profile (shown in Fig. 9c) is estimated from Raman lidar-measured aerosol-scattering ratio profile, for which the total extinction is normalized by the MFRSR-measured aerosol optical depth (shown in Fig. 8b).

The comparisons of measured and simulated direct-beam spectra at the oxygen A-band under different solar zenith angles (SZA) are shown in Fig. 10. In this study, we use relative difference to quantify the comparison, which is indicated by the ratio of the absolute difference to the observation value. The maximum relative difference between them often occurs at or near strong absorption line centers, which is very sensitive to wavelength shift and has low SNR (Fig. 7b). At small SZA (e.g., SZA $=27^{\circ}$ ), the confidence interval $(95 \%)$ of relative difference is $(-0.06,0.05)$. At large SZA (e.g., $\mathrm{SZA}=72^{\circ}$ ), the SNR at or near strong absorption line centers decreases significantly. The confidence interval of relative difference is $(-0.08,0.09)$. Figure 11 shows the comparisons of normalized direct-beam radiance of the oxygen A-band spectra versus air mass. In this study, the normalized radiances at five wavelengths are chosen for the analysis (shown in Fig. 11b). In general, the absolute difference of normalized radiance between observation and simulation is small. The HABS direct-beam measurements and model simulations are basically consistent with each other for different air masses. Meanwhile, for any given wavelength position, the logarithmic values of normalized radiance decreases quasi-linearly with air mass. The slight non-linear part could 

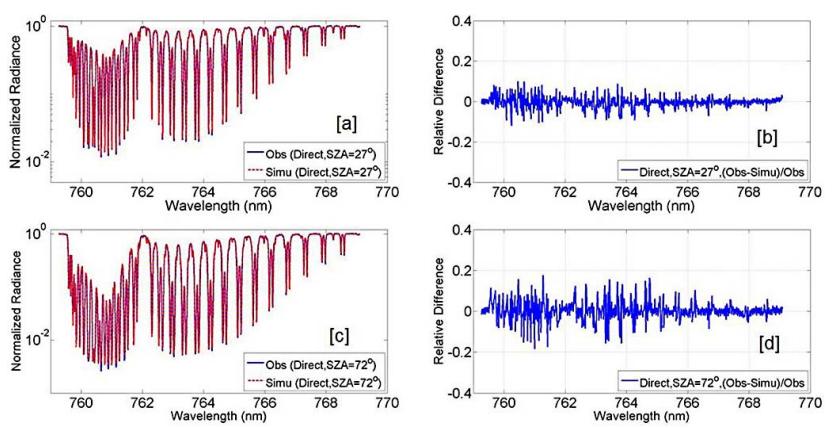

Figure 10. Comparisons of normalized radiance of HABSmeasured and model simulated direct-beam spectra at oxygen Aband under different solar zenith angles.

be caused by several reasons: (1) strong oxygen absorption; (2) temporal variation of atmospheric profiles (e.g., temperature profile, pressure profile, and aerosol profile); and (3) measurement errors associated with the wavelength registration and elevation-azimuth sun tracker accuracy.

Figure 12 shows the comparisons of measured and simulated zenith diffuse spectra at the oxygen A-band for different SZAs. The measured zenith diffuse spectra used for comparison are combined by the measurements from 4 polarizer channels (e.g., $I\left(0^{\circ}\right)+I\left(90^{\circ}\right)$ or $\left.I\left(45^{\circ}\right)+I\left(135^{\circ}\right)\right)$. The combined measured spectra have the ability to remove or constrain the impacts of the instrument polarization performance. The difference of normalized radiance between observation and simulation is also quite small. The relative difference of diffuse spectra between observation and simulation is similar to that of direct-beam spectra. With SZA of 27 and $72^{\circ}$, the confidence intervals of relative differences are $(-0.06,0.05)$ and $(-0.08,0.07)$, respectively. In particular, in the wavelength range around the strong oxygen absorption line centers (e.g., range of 763 to $765 \mathrm{~nm}$ ), we found that the simulated value at the absorption line centers tends to be slightly smaller than observation. This could be caused by two factors: (1) the error of instrument slit function measurement and oxygen absorption line parameters; and (2) Raman scattering effects (Sioris and Evans, 2000). For the highresolution diffuse-radiation spectrum, Raman scattering occurs in 3 to $5 \%$ of the Rayleigh scattering events. It slightly fills in $\mathrm{O}_{2}$ A-band, especially at the $\mathrm{O}_{2}$ absorption line centers. However, the Raman scattering effect is not treated in the current version of HABS simulator, and it will be analyzed in detail in our further studies. In general, the HABS observation and the related model simulation of zenith diffuse radiance at the oxygen A-band are basically consistent with each other.

The relative difference between measurements and model simulation, to some extent, always exists for both direct beam and zenith diffuse radiance. To reduce the difference between observation and simulation for radiation closure, further studies are needed: (1) to have independent measurements of
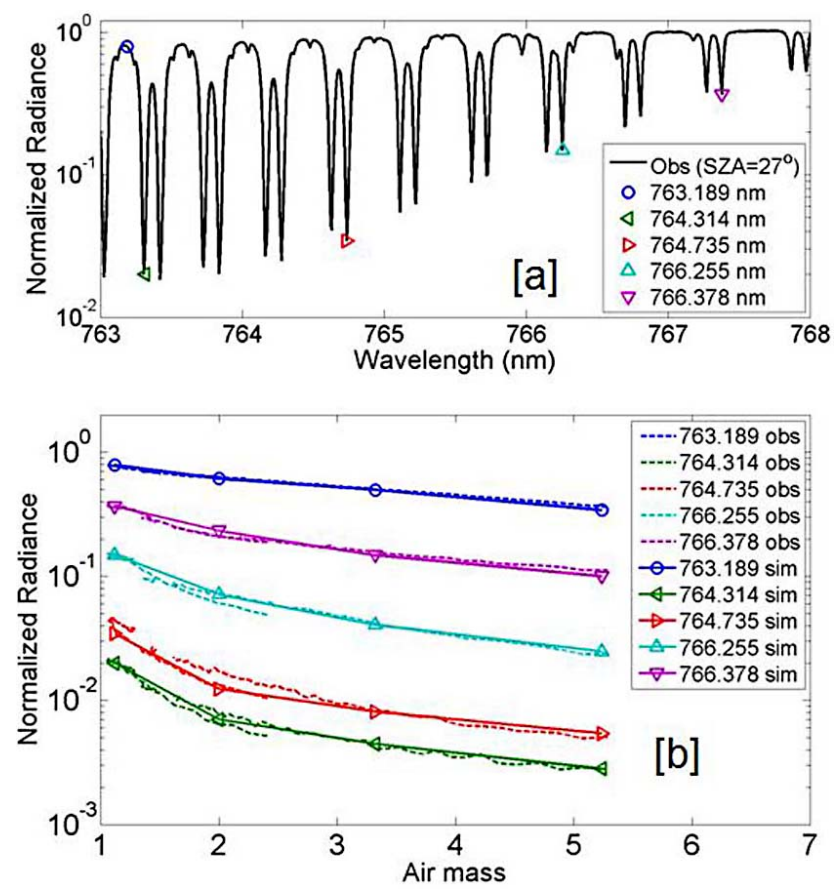

Figure 11. Comparisons of normalized radiance of HABSmeasured and model simulated direct-beam spectra at oxygen Aband versus air mass.

high-resolution $\mathrm{O}_{2}$ A-band spectrum for cross validating the measurement accuracy; (2) to further evaluate and improve the instrument slit function and performance; (3) to perfect the oxygen absorption line parameters; (4) to improve the radiative transfer model and accuracy of input parameters; and (5) to consider other issues, such as Raman scattering effect.

\section{Discussion and applications of HABS}

This HABS provides accurate high-resolution radiance and polarization spectra of both direct beam and zenith diffuse with large dynamic range at oxygen A-band, as shown in Figs. 6 and 7. The concept underlying all oxygen A-band retrievals is the principle of equivalence (Irvine, 1964, 1966; van de Hulst, 1980). As a hidden property of standard radiation transfer theory and controlled by spatial distributions of scattering and absorption, photon path length distributions can be retrieved through an inverse Laplace transform based on the equivalence theorem. Because the scattering properties of cloud and aerosol vary slowly and predictably with wavelength and the oxygen A-band is located at a central wavelength of solar shortwave spectrum, the photon path length distribution retrieved from oxygen A-band spectrometry is reasonably representative of the entire solar shortwave. Given the performance of HABS, about four independent pieces of information of photon path length distribution can be retrieved from the measured spectra (Min and Harrison, 

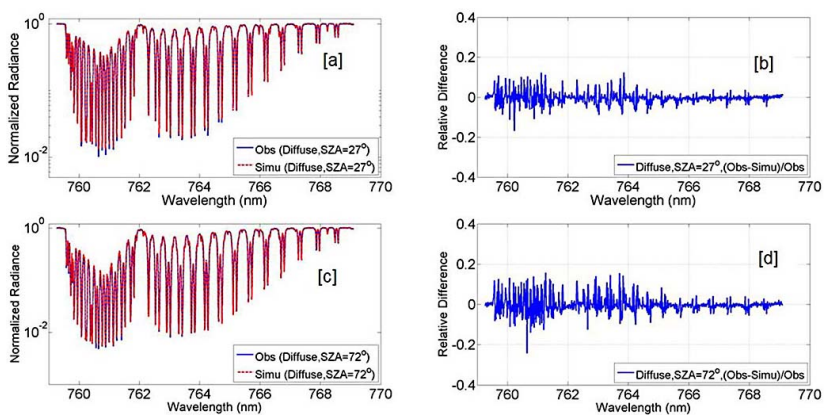

Figure 12. Comparisons of measured and simulated diffuse zenith sky spectra at oxygen A-band for solar zenith angles of $27^{\circ}$ (a and b) and $72^{\circ}$ (c and d).

2004). The photon path length distributions, the fundamental application of HABS measurements, provide the vertical information of scattering and absorption in the atmosphere.

Detailed knowledge of the vertical distribution of cloud macrophysical/microphysical properties is important to calculate the Broadband Heating Rate Profile, a fundamental driver in climate system (Stephens, 1978; Li and Min, 2010). However, our current remote-sensing methods for retrieving cloud properties are still uncertain, particularly under 3-D cloud conditions. Li and Min (2010) illustrated that the variance and mean of photon path length from the modest resolution oxygen A-band spectral measurements of rotating shadowband spectrometer (RSS) is sensitive to detect possible "missed" upper layer clouds by a collocated high-sensitivity millimeter wavelength cloud radar (MMCR), that is, at least $27 \%$ of single-layer clouds detected by MMCR radar could be multi-layer clouds or influenced by 3-D effects. Although "missed" cloud layer does not occur all the time, statistical information of "missed" cloud layer is extremely valuable for calculating a broadband heating rate profile. Furthermore, at present validation of broadband heating rate profile and various cloud overlap schemes in GCMs is limited to comparison with the observed cloud cover and radiation fluxes at the top of the atmosphere and at the surface. The representativeness of various statistics of cloud geometry has not yet been tested against observations, particularly the heating profiles associated with vertical distribution of clouds. The closures of radiation fluxes at the top of the atmosphere and at the surface do not ensure the accuracy of the heating profile. The lack of rigorous validation makes it difficult to assess the relative merits of different cloud overlap treatments and broadband heating rate profile products. Photon path length distributions inferred from HABS measurements provide the links between cloud microphysics and 3D cloud geometry measured from 3-D scanning radars and lidars to understand the 3-D radiative transfer in the atmosphere and its impacts on remote sensing. Higher moments of photon path length distribution derived from high-resolution oxygen A-band spectrometer of HABS will provide vertical constraints to ensure the accuracy of heating profiles. Therefore, we will further exploit the HABS applications through the joint statistic approach (photon path length and optical properties derived from radiance/irradiance at non-absorbing wavelengths), which provides a unique means to detect the 3$\mathrm{D}$ structures of clouds and aerosols and to validate broadband heating profiles and GCM overlap schemes in both instantaneous cases and statistical ensembles (Min and Harrison, 1999; Min et al., 2001; Min and Clothiaux, 2003).

The current remote-sensing techniques are unable to detect all relevant information to derive cloud microphysical and optical properties. All retrievals from those measurements with limited information have to make some assumptions. Those assumptions may introduce some uncertainties. An important challenge is to reduce ambiguity and uncertainty by minimizing and/or constraining those assumptions in retrievals. It is advantageous to combine HABS with other sensors, in particular, with active lidar and radar systems to enhance the information content. For example, cloud radar signal is capable of penetrating clouds and revealing multiple layers aloft. However, the observed radar reflectivity is a function of the sixth moment of the hydrometeor particle-size distribution. It requires an assumption of particle-size distribution to retrieve cloud water content (the third moment of the hydrometeor particle-size distribution) for practical applications from radar reflectivity. As the pressure dependence of oxygen A-band absorption line parameters provides vertical resolving power and photon path length information inferred from oxygen A-band spectra is a function of the second moment of particle-size distribution, HABS-measured oxygen A-band spectra can be used to better constrain the assumed particle-size distribution of radar retrievals, particularly for multi-layer clouds and for mixed-phase clouds ( $\mathrm{Li}$ and Min, 2013). Since HABS provides at least four additional pieces of independent information on cloud-aerosol properties, we would expect to get more accurate cloud and aerosol microphysical/optical profiles than the current existed retrievals. With the polarization capability of HABS, combining of HABS observations with lidar measurements provides better retrievals of vertical profiles of aerosols and thin clouds, particularly thin ice clouds.

HABS has another important application to provide the "ground true" for the space-based $\mathrm{O}_{2}$ A-band observation. Due to photon multiple scattering and absorption inside cloud and aerosol layers, the transmittance and reflectance across $\mathrm{O}_{2}$ A-band spectrum have different sensitivity with respect to cloud-aerosol optical property and vertical distribution and to surface albedo. Therefore, the transmitted $\mathrm{O}_{2} \mathrm{~A}$ band spectrum observed by HABS is complement of the reflected $\mathrm{O}_{2} \mathrm{~A}$-band spectrum by space-based sensors, in terms of radiation transfer in the atmosphere. The observed radiances from surface HABS not only provide the basis for evaluation and validation of spaced-based $\mathrm{O}_{2}$ A-band observation, but also enable one to understand the radiation transfer and closure in the atmosphere through the combination of 
transmitted and reflected $\mathrm{O}_{2}$ A-band observations. Particularly, the polarization capability of HABS enables the evaluation of the high-resolution $\mathrm{O}_{2} \mathrm{~A}$-band measurements on Orbiting Carbon Observatory-2 (OCO-2) satellite, which only measures one component of polarization of $\mathrm{O}_{2}$ A-band spectrum.

\section{Summary}

The newly developed high-resolution A-band spectrometer (HABS) was evaluated with in-lab tests and through deployment during the NASA Discover-AQ field campaign at HUBC in July 2011. The HABS exhibits excellent performance: stable spectral response ratio, high SNR of 100 at the darkest pixels in the $\mathrm{P}$ branch, high-spectrum resolution of $0.016 \mathrm{~nm}$ or $0.26 \mathrm{~cm}^{-1}$, and high out-of-band rejection of $10^{-5}$. With such specifications, the HABS-measured oxygen A-band spectra is able to derive at least four independent pieces of information (Min and Harrison, 2004). With an altazimuth tracker fore-optics, the HABS measures both direct beam and diffuse radiances with the same system, enabling better assessments of instrument functions and absorption line parameters and construction of retrieval kernel functions (Min and Clothiaux, 2003). More importantly, the HABS has the capability to measure the polarization of atmospheric scattering, providing additional information for scattering particle shapes.

The HABS spectra measurements and model simulations are basically consistent with each other. The significant/large relative differences between them mainly occur at or near the strong absorption line centers, which increase with the SZA. For the direct-beam spectra, the confidence intervals $(95 \%)$ of relative difference between measurements and simulations are $(-0.06,0.05)$ and $(-0.08,0.09)$ for SZAs of 27 and $72^{\circ}$, respectively. For the zenith diffuse spectra, the confidence intervals of relative difference are $(-0.06,0.05)$ and $(-0.08,0.07)$ for SZAs of 27 and $72^{\circ}$, respectively. In particular, for large SZAs, the simulated zenith diffuse radiance at the absorption line centers tends to be slightly smaller than observed one.

A high-resolution A-band spectrometer is unique and capable instrument for cloud and aerosol detection in the real 3D atmosphere. In the near future, we will (1) further assess and improve the HABS performance; (2) improve the radiative transfer model, including the Raman scattering effect; (3) retrieve the photon path length distribution; (4) explore the polarization capability of HABS for retrieving the optical properties of aerosols and thin clouds; (5) combine the HABS measurements with active sensor (radar and lidar) to retrieve the vertical profiles of aerosol and cloud optical property; and (6) validate the satellite measurements and further understand 3-D radiation transfer in the atmosphere.
Acknowledgements. This work was supported by US DOE's Atmospheric System Research program (Office of Science, OBER) under contract DE-FG02-03ER63531, by the NSF under contract AGS-1138495, and by the NOAA Educational Partnership Program with Minority Serving Institutions (EPP/MSI) under cooperative agreements NA17AE1625 and NA17AE1623. Part of this research was also supported by Strategic Priority Research Program of the Chinese Academy of Sciences (XDA05040300) and National High Technology Research and Development Program of China (no. 2011AA12A104).

Edited by: M. Wendisch

\section{References}

Berry, H. G., Gabrielse, G., and Livingston, A. E.: Measurement of the stokes parameters of light, Appl. Optics, 16, 3200-3205, doi:10.1364/AO.16.003200, 1977.

Boesche, E., Stammes, P., Ruhtz, T., Preusker, R., and Fischer, J.: Effect of aerosol microphysical properties on polarization of skylight: sensitivity study and measurements, Appl. Optics, 45, 8790-8805, 2006.

Boesche, E., Stammes, P., and Bennartz, R.: Aerosol influence on polarization and intensity in near-infrared $\mathrm{O}_{2}$ and $\mathrm{CO}_{2}$ absorption bands observed from space, J. Quant. Spectrosc. Ra., 110, 223-239, 2009.

Bréon, F. M. and Colzy, S.: Global distribution of cloud droplet effective radius from POLDER polarization measurements, Geophys. Res. Lett., 27, 4065-4068, 2000.

Chance, K. and Kurucz, R. L.: An improved high-resolution solar reference spectrum for Earth's atmosphere measurements in the ultraviolet, visible, and near infrared, J. Quant. Spectrosc. Ra., $111,1289-1295,2010$

Charles, N. A. and Kattawar, G. W.: Effect of volume-scattering function on the errors induced when polarization is neglected in radiance calculations in an atmosphere-ocean system, Appl. Optics, 32, 4610-4617, 1994.

Chou, M. D. and Kouvaris, L.: Monochromatic calculation of atmospheric radiative transfer due to molecular line absorption, J. Geophys. Res., 91, 4047-4055, 1986.

Chowdhary, J., Cairns, B., Mishchenko, M., and Travis, L.: Retrieval of aerosol properties over the ocean using multispectral and multiangle photopolarimetric measurements from the Research Scanning Polarimeter, Geophys. Res. Lett., 28, 243-246, 2001.

Chowdhary, J., Cairns, B. Mishchenko, M. I., Hobbs, P. V., Cota, G. F., Redemann, J., Rutledge, K., Holben, B. N., and Russell, E.: Retrieval of aerosol scattering and absorption properties from photopolarimetric observations over the ocean during the CLAMS experiment, J. Atmos. Sci., 62, 1093-1117, 2005.

Clough, S. A., Shephard, M. W., Mlawer, E. J., Delamere, J. S., Iacono, M. J., Cady-Pereira, K., Boukabara, S., and Brown, P. D.: Atmospheric radiative transfer modeling: a summary of the AER codes, Short Communication, J. Quant. Spectrosc. Ra., 91, 233-244, 20005.

Connell, R. M., Adam, M., and Venable, D.: A numerical model of the performance of the Howard University Raman Lidar System, AIP Conf. Proc., 1140, 99-103, doi:10.1063/1.3183533, 2009. 
Deuzé, J. L., Goloub, P., Herman, M., Marchand, A., Perry, G., Susana, S., and Tanré, D.: Estimate of the aerosol properties over the ocean with POLDER, J. Geophys. Res., 105, 15329-15346, 2000.

Deuzé, J. L., Bréon, F. M., Devaux, C., Goloub, P., Herman, M., Lafrance, B., Maignan, F., Marchand, A., Nadal, F., Perry, G., and Tanré, D.: Remote sensing of aerosols over land surfaces from POLDER-ADEOS-1 polarized measurements, J. Geophys. Res., 106, 4913-4926, 2001.

Duan, M., Min, Q., and Li, J.: A fast radiative transfer model for simulating high-resolution absorption bands, J. Geophys. Res., 110, D15201, doi:10.1029/2004JD005590, 2005.

Fischer, J. and Grassl, H.: Detection of cloud-top height from backscattered radiances within the oxygen A band. Part I: Theoretical study, J. Appl. Meteorol., 30, 1245-1259, 1991.

Fischer, J., Cordes, W., Schmitz-Peiffer, A., Renger, W., and Morel, P.: Detection of cloud-top height from backscattered radiances within the oxygen A band. Part II: Measurements, J. Appl. Meteorol., 30, 1260-1267, 1991.

Grechko, Y. I., Dianov-Klokov, V. I., and Malkov, I. P.: Aircraft measurements of photon paths in reflection and transmission of light by clouds in the 0.76 um oxygen band, Atmos. Ocean. Phys., 9, 471-485, 1973.

Hansen, J. E. and Travis, L. D.: Light scattering in planetary atmospheres, Space Sci. Rev., 16, 527-610, 1974.

Harrison, L. and Min, Q.-L.: Photon Pathlength Distributions in Cloudy Atmospheres From Ground-based High-resolution $\mathrm{O}_{2}$ A-band Spectroscopy, in IRS'96: Current Problems in Atmospheric Radiation, edited by: Smith, W. L. and Stamnes, K., Deepak Pub, Hampton, 594-602, 1997.

Heidinger, A. and Stephens, G. L.: Molecular line absorption in a scattering atmosphere, 2: Retrieval of particle properties, J. Atmos. Sci., 57, 1615-1634, 2000.

Heidinger, A. and Stephens, G. L.: Molecular line absorption in a scattering atmosphere, 3: Path length characteristics and effects of spatially heterogeneous clouds, J. Atmos. Sci., 59, 1641-1654, 2002.

Irvine, W. M.: The formation of absorption bands and the distribution of photon optical paths in a scattering atmosphere, Bull. Astron. Inst. Neth., 17, 266-279, 1964.

Irvine, W. M.: The shadowing effect in diffuse, J. Geophys. Res., 71, 2931-2937, doi:10.1029/JZ071i012p02931, 1966.

Koelemeijer, R. B., Stammes, P., Hovenier, J. W., and Haan, J. D.: A fast method for retrieval of cloud parameters using oxygen A band measurements from the Global Ozone Monitoring Experiment, J. Geophys. Res.-Atmos., 106, 3475-3490, 2001.

Kokhanovsky, A. A., Rozanov, V. V., Nauss, T., Reudenbach, C., Daniel, J. S., Miller, H. L., and Burrows, J. P.: The semianalytical cloud retrieval algorithm for SCIAMACHY I. The validation, Atmos. Chem. Phys., 6, 1905-1911, doi:10.5194/acp-6-1905-2006, 2006a.

Kokhanovsky, A. A., von Hoyningen-Huene, W., Rozanov, V. V., Noël, S., Gerilowski, K., Bovensmann, H., Bramstedt, K., Buchwitz, M., and Burrows, J. P.: The semianalytical cloud retrieval algorithm for SCIAMACHY II. The application to MERIS and SCIAMACHY data, Atmos. Chem. Phys., 6, 4129-4136, doi:10.5194/acp-6-4129-2006, 2006b.

Kuze, A., Suto, H., Nakajima, M., and Hamazaki, T.: Thermal and near infrared sensor for carbon observation Fourier-transform spectrometer on the Greenhouse Gases Observing Satellite for greenhouse gases monitoring, Appl. Optics, 48, 6716-6733, 2009.

Lacis A. A., Chowdhary, J., Mishchenko, M. I., and Cairns, B.: Modeling errors in diffuse-sky radiation: vector versus scalar treatment, J. Geophys. Res., 25, 135-138, 1998.

Levy, R. C., Remer, L. A., and Kaufman, Y. J.: Effects of neglecting polarization on the MODIS aerosol retrieval over land, IEEE Geosci. Remote Sens., 42, 2576-2583, 2004.

Li, S. and Min, Q.: Diagnosis of multilayer clouds using photon path length distributions, J. Geophys. Res., 115, D20202, doi:10.1029/2009JD013774, 2010.

Li, S. and Min, Q.: Wavelength registration of high resolution oxygen A-band spectral measurements, J. Quant. Spectrosc. Ra., 122, 106-123, doi:10.1016/j.jqsrt.2012.10.024, 2012.

Li, S. and Min, Q.: Retrievals of vertical profiles of stratus cloud properties from combined oxygen A-band and radar observations, J. Geophys. Res. Atmos., 118, 769-778, doi:10.1029/2012JD018282, 2013.

McMaster, W. H.: Polarization and the Stokes parameters, Am. J. Phys., 22, 351-362, doi:10.1119/1.1933744, 1954.

McMaster, W. H.: Matrix representation of polarization, Rev. Mod. Phys., 8, 33, 8-28, doi:10.1103/RevModPhys.33.8, 1961.

Min, Q.-L. and Clothiaux, E. E.: Photon path length distributions inferred from rotating shadowband spectrometer measurements at the Atmospheric Radiation Measurements Program Southern Great Plains site, J. Geophys. Res., 108, 4465, doi:10.1029/2002JD002963, 2003.

Min, Q.-L. and Harrison, L. C.: Cloud properties derived from surface MFRSR measurements and comparison with GOES results at the ARM SGP site, Geophys. Res. Lett., 23, 1641-1644, 1996.

Min, Q.-L. and Harrison, L. C.: Joint statistics of photon path length and cloud optical depth, Geophys. Res. Lett., 26, 1425-1428, 1999.

Min, Q.-L. and Harrison, L. C.: Retrieval of atmospheric optical depth profiles from downward-looking high-resolution O2 Aband measurements: optically thin conditions, J. Atmos. Sci., 61, 2469-2478, 2004.

Min, Q.-L., Harrison, L. C., and Clothiaux, E.: Joint statistics of photon pathlength and cloud optical depth: case studies, J. Geophys. Res., 106, 7375-7386, 2001.

Min, Q.-L., Duan, M., and Marchand, R.: Validation of surface retrieved cloud optical properties with in situ measurements at the Atmospheric Radiation Measurement Program (ARM) South Great Plains site, J. Geophys. Res., 108, 4547, doi:10.1029/2003JD003385, 2003.

Min, Q.-L., Harrison, L. C., Kierdron, P., Berndt, J., and Joseph, E.: A high-resolution oxygen A-band and water vapor band spectrometer, J. Geophys. Res., 109, D02202, doi:10.1029/2003JD003540, 2004a.

Min, Q.-L., Joseph, E., and Duan, M.: Retrievals of thin cloud optical depth from a multifilter rotating shadowband radiometer, J. Geophys. Res., 109, D02201, doi:10.1029/2003JD003964, 2004b.

Min, Q.-L., Minnis, P., and Khaiyer, M.: Comparison of cirrus optical depths derived from GOES 8 and surface measurements, J. Geophys. Res., 109, D15207, doi:10.1029/2003JD004390, 2004c. 
Min, Q.-L., Wang, T., Long, C. N., and Duan, M.: Estimating fractional sky cover from spectral measurements, J. Geophys. Res., 113, D20208, doi:10.1029/2008JD010278, 2008.

Mishchenko, M. I., Lacis, A. A., and Travis, L. D.: Errors induced by the neglect of polarization in radiance calculations for Rayleigh-scattering atmospheres, J. Quant. Spectrosc. Ra., 51, 491-510, 1994.

Mitchell, R. M. and O'Brien, D. M.: Error estimates for passive satellite measurement of surface pressure using absorption in the A band of oxygen, J. Atmos. Sci., 44, 1981-1990, 1987.

Natraj, V., Spurr, R., Boesch, H., Jiang, Y., and Yung, Y.: Evaluation of errors in neglecting polarization in the forward modeling of $\mathrm{O}_{2} \mathrm{~A}$ band measurements from space, with relevance to $\mathrm{CO}_{2}$ column retrieval from polarization sensitive instruments, J. Quant. Spectrosc. Ra., 103, 245-259, 2007.

O'Brien, D. M. and Mitchell, R. M.: Error estimates for retrieval of cloud top pressure using absorption in the A band of oxygen, J. Appl. Meteor., 31, 1179-1192, 1992.

Oikarinen, L.: Polarization of light in UV-visible limb radiance measurements, J. Geophys. Res., 106, 1533-1544, 2001.

Pfeilsticker, K., Erle, F., Veitel, H., and Platt, U.: First Geometrical pathlengths probability density function derivation of the skylight from spectroscopically highly resolving oxygen A-band observations: 1. Measurement technique, atmospheric observations and model calculations, J. Geophys. Res., 103, 11483-11504, 1998.

Platt, U. and Stutz, J.: Differential Optical Absorption Spectroscopy: Principles and Applications, Springer Verlag, Heidelberg, ISBN 978-3540211938, 597 pp., 2008.

Portmann, R. W., Solomon, S., Sanders, R. W., and Danel, J. S.: Cloud modulation of zenith sky oxygen path lengths over Boulder, Colorado: Measurements versus model, J. Geophys. Res., 106, 1139-1155, 2001.

Rodgers, C. D.: Inverse Methods for Atmospheric Sounding: Theory and Practice, World Scientific, 2000.

Rothman, L. S., Gordon, I. E., Barbe, A., Benner, D. C., Bernath, P. F., Birk, M., Boudon, V., Brown, L. R., Campargue, A., Champion, J.-P., Chance, K., Coudert, L. H., Dana, V., Devi, V. M., Fally, S., Flaud, J.-M., Gamache, R. R., Goldman, A., Jacquemart, D., Kleiner, I., Lacome, N., Lafferty, W. J., Mandin, J.-Y., Massie, S. T., Mikhailenko, S. N., Miller, C. E., Moazzen-Ahmadi, N., Naumenko, O. V., Nikitin, A.V., Orphal, J., Perevalov, V. I., Perrin, A., Predoi-Cross, A., Rinsland, C. P., Rotger, M., Simeckova, M., Smith, M. A. H., Sung, K., Tashkun, S. A., Tennyson, J., Toth, R. A., Vandaele, A. C., and Auwera, J. V.: The HITRAN 2008 molecular spectroscopic database, J. Quant. Spectrosc. Ra., 110, 533-572, 2009.
Schutgens, N. A. J. and Stammes, P.: A novel approach to the polarization correction of spaceborne spectrometers, J. Geophys. Res., 108, 4229, doi:10.1029/2002JD002736, 2003.

Sioris, C. E. and Evans, W. F.: Impact of rotational Raman scattering in the O2A band, Geophys. Res. Lett., 27, 4085-4088, 2000.

Spiering, F. R., Kiseleva, M. B., Filippov, N. N., Naus, H., Lieshout, B. V., Weijenborg, C., and Zande, W. J.: Line mixing and collision induced absorption in the oxygen A-band using cavity ring-down spectroscopy, J. Chem. Phys., 133, 114305, doi:10.1063/1.3460924, 2010.

Stam, D. M. and Hovenier, J. W.: Errors in calculated planetary phase functions and albedos due to neglecting polarization, Astron. Astrophys., 444, 275-286, 2005.

Stam, D. M., De Haan, J. F., Hovenier, J. W., and Stammes, P.: Degree of linear polarization of light emerging from the cloudless atmosphere in the oxygen A band, J. Geophys. Res.-Atmos., 104, 16843-16858, 1999.

Stamnes, K., Tsay, S. C., Jayaweera, K., and Wiscombe, W.: Numerically stable algorithm for discrete ordinate method radiative transfer in multiple scattering and emitting layered media, Appl. Optics, 27, 2502-2509, 1988.

Stephens, G. L.: Radiation profiles in extended water clouds. I: Theory, J. Atmos. Sci., 35, 2111-2122, 1978.

Stephens, G. L. and Heidinger, A.: Molecular line absorption in a scattering atmosphere: I Theory, J. Atmos. Sci., 57, 1599-1614, 2000.

Stephens, G. L., Heidinger, A., and Gabriel P.: Photon paths and cloud heterogeneity: Toward an observational strategy for assessing the effects of 3D geometry on radiative transfer, ThreeDimensional Radiative Transfer for Cloudy Atmospheres, 2005.

Van de Hulst, H. C.: Scattering of light by small particles, Moscow: Izd-vo inostr. lit., 1957.

Van de Hulst, H. C.: Multiple Light Scattering, Tables, Formulas and Applications, Vol. 2, Academic, London, 1980.

Veitel, H., Funk, O., Kurz, C., Platt, U., and Pfeilsticker, K.: Geometrical pathlength probability density functions of the skylight transmitted by midlatitude cloudy skies: some case studies, Geophys. Res. Lett., 25, 3355-3358, 1998. 